\title{
Novel Approaches to Vasculopathies: The Role of Stem Cells and Regenerative Medicine
}

\section{Jason Seewoodhary}

Specialist Registrar in Diabetes Mellitus \& Endocrinology / General Internal Medicine,UK

*Corresponding author: Dr. Jason Seewoodhary, Specialist Registrar, Department of Diabetes Mellitus and Endocrinology, Wrexham Maelor Hospital, Croesnewydd Road, Wrexham, LL13 7TD, United Kingdom, Tel: +44 (0)7908070455; E-mail: seewoodharyj@hotmail.com

Received date: May 14, 2014, Accepted date: July 14, 2014, Published date: July 21, 2014

Copyright: (c) 2014 Jason Seewoodhary. This is an open-access article distributed under the terms of the Creative Commons Attribution License, which permits unrestricted use, distribution, and reproduction in any medium, provided the original author and source are credited.

\begin{abstract}
The potential utility of stem cell therapies for vascular regeneration provides a novel disease-modifying approach towards vasculopathies such as coronary artery disease, peripheral vascular disease and diabetic retinopathy. Stem cell therapies can potentially replace damaged vascular tissue, attenuate further vascular damage, and release soluble factors that act in a paracrine or endocrine manner to facilitate repair and reversal of the pathology that underlies the genesis and propagation of damage within the vasculature. This review critically considers the role of stem cell therapeutics in vascular regeneration.
\end{abstract}

Keywords: Stem Cells; Vasculogenesis; Angiogenesis; Arteriogenesis; Regeneration

\begin{abstract}
Abbreviations
ASTAMI: Autologous Stem cell Transplantation in Acute Myocardial Infarction; BAMI: Bone marrow-derived mononuclear stem cells (BM-MNC) on all-cause mortality in Acute Myocardial Infarction; bFGF: basic Fibroblast Growth Factor; BM-MNC: Bone marrow-derived mononuclear stem cells; BMP-4: Bone Morphogenetic Protein-4; BOOST: BOne marrOw cell transfer in patients with acute ST-elevation myocardial infarction; CXCR-4: Chemokine Receptor type-4; EPC: Endothelial Progenitor Cell; ES: Embryonic Stem; G-CSF: Granulocyte Colony Stimulating Factor; iPS: Induced Pluripotent Stem; LVEF: Left Ventricular Ejection Fraction; MSC: Mesenchymal Stem Cell; NSC: Neural Stem Cell; PiPSC's: Protein-Induced Pluripotent Stem Cells; REPAIR-AMI: Reinfusion of Enriched Progenitor cells And Infarct Re-modelling in Acute Myocardial Infarction; SCID: Severe Combined Immunodeficiency; SDF-1 $\alpha$ : Stromal Cell Derived Factor-1a; SVZ: Sub-Ventricular Zone; TACT: Therapeutic Angiogenesis using Cell Transplantation; TransACT-1 and 2: Transplantation of enriched autologous bonemarrow derived $\mathrm{CD} 133+$ cells in patients having coronary surgery after STEMI 1 and 2 trial; VEGF: Vascular Endothelial Growth Factor; WHO: World Health Organisation
\end{abstract}

\section{Introduction}

According to the World Health Organization (WHO) cardiovascular disease is the number one cause of death globally. In particular, the Global Burden of Disease study classified ischemic heart disease as the leading cause of global mortality, accounting for 1.4 million deaths in the developed world and 5.7 million deaths in developing regions [1]. Vascular disease, senescence and dysfunction is central to the pathogenesis of cardiovascular disease. Accordingly, much attention has shifted towards the therapeutic role of vascular regeneration, which encompasses vasculogenesis, angiogenesis and arteriogenesis and refers to the formation and renewal of normal vascular structure and function. The aim of vascular regeneration is to guard against vascular senescence, which is associated with: ischemic end-organ damage due to hypoxia, reperfusion or capillary leak; and complications such as thromboembolism, plaque rupture or dissection. Exploiting the potential utility of vascular regenerative therapies may shift treatment paradigms for vascular diseases towards curation. This review will critically consider the role of stem cells for vascular regeneration.

\section{Stem Cells}

Stem cells are undifferentiated cells capable of unlimited proliferation and self-renewal whilst retaining the potential towards differentiation into any cell type of endodermal, ectodermal or mesodermal origin. There are three main types of stem cells: Embryonic Stem- (ES) cells, adult stem cells, and induced Pluripoetnt Stem- (iPS) cells.

\section{Embryonic Stem Cells}

ES cells are pluripotent cells derived from the inner cell mass of the developing blastocyst. ES cells confer the advantage of being: renewable; accessible to genetic modifications; and expandable in vitro for lengthy periods. Thus ES cells can be yielded in high purified quantities for potential regenerative purposes. Disadvantages of ES cells include: a relatively high tumourigenic potential; transplant rejection; and ethical concerns relating to disaggregating the developing blastocyst.

\section{Adult Stem Cells}

Adult stem cells are multipotent undifferentiated cells. They are derived from specific tissues within the embryo, foetus or adult e.g. the Subventricular Zone (SVZ), which contains Neural Stem Cells (NSC's), or the bone marrow, which contains two types of adult stem cells, namely, Mesenchymal Stem Cells (MSC's) and haematopoietic stem cells. Advantages of adult stem cells include self-renewability and the potential to be harvested from easily accessible organs and 
expanded. Furthermore, relative to ES cells, adult stem cells have a superior safety profile with a lower tumorigenic potential and incur fewer ethical issues. Compared to ES cells disadvantages include a lower degree of plasticity, expandability, and renewability, coupled with a greater susceptibility to senescence. Other limitations include invasive harvesting methods e.g. bone marrow trephine and biopsy to obtain MSC's. Furthermore, in contrast to ES cells, adult stem cells are rarer in number in mature tissues, which is significant as large numbers of cells are needed for stem cell replacement therapies.

\section{Induced Pluripotent Stem Cells}

iPS cells are derived from non-pluripotent somatic cells such as dermal fibroblasts, which have been transformed and genetically 'reprogrammed' into a pluripotent state. This is achieved by transfection with transcription factors such as Oct-3/4, Sox 2 and Nanog, which are core transcription factors that repress the expression profile of differentiated cells and activate an array of genes involved in pluripotency. Other key transcription factors include Klf-4, Lin28 and $c-M y c$. Four traditional strategies are available to reprogramme somatic cells to an iPS cell state: viral transduction; nuclear transfer; cell fusion; and cell explantation. iPS cells offer the advantage of being: easily and non-invasively harvested; useful tools for drug development; models for disease processes in vitro; and a source of autologous cells for transplantation due to a lower risk of immunorejection. Disadvantages include a propensity towards tumorigenesis and a lack of long-term data on stability and safety.

Limitations of the transcription factor approach to make iPS cells include: a low throughput; mutations being inserted into the target cells genome; tumours, especially with $c-M Y C$; and incomplete reprogramming. These limitations can be overcome by novel techniques to make iPS cells, which include: ES cell specific microRNA's (miRNA's) to prompt iPS cell reprogramming; using biomimicry with recombinant proteins injected into cells via polyarginine anchors, which has coined the nomenclature of piPSCsprotein-induced pluripotent stem cells; and small compound mimicking, which raises reprogramming efficiency.

\section{Embryonic Stem Cell Treatments}

ES cells can be differentiated into cardiomyocytes, smooth muscle-, and Endothelial Progenitor Cells (EPC's). ES cell-derivatives have an evidence base to support their utility in the treatment of vascular regeneration; labelled mouse ES cell lines that were grafted into rodent models of myocardial infarction and tracked using histochemistry and immunofluorescence were found to differentiate into EPC's, cardiomyocytes, and smooth muscle cells and improve cardiac function [2]. The mechanism of benefit was demonstrated by evidence from a separate study using sequential echocardiography and detailed histological analysis. Engrafted human ES cell-derived cardiomyocytes were reported to attenuate pathological remodelling and preserve regional and global contractile function by improving fractional shortening, left ventricular diastolic dimensions and the wall motion score index in infarcted rat hearts [3]. However, approaches to generate human ES-derived cardiomyocytes were limited by inefficient differentiation and purity methods and poor graft survival posttransplantation. This has since been overcome by novel refined laboratory techniques that have enabled the generation of highly purified human cardiomyocytes using a scalable system for directed differentiation using Bone Morphogenic Protein-4 (BMP-4) and activin A [4]. Recent evidence suggests a crucial role of extracellular matrix signalling in determining cell lineage commitment for cardiovascular tissue engineering [5]. Further corroboratory evidence using a non-human primate model of myocardial ischaemia followed by reperfusion demonstrated that cryopreservation and intramyocardial delivery of one billion human ES cell-derived cardiomyocytes generated extensive remuscularisation of the infarcted heart [6].

In addition to ES cell-derived cardiomyocytes, ES cell derivedEPC's also have a demonstrable efficacy for vascular regeneration. In a rodent model of myocardial infarction it was shown that bioluminescent labelled ES cell-derived EPC's and mural cells enhance myocardial function and contractility following injection into the ischemic anterior wall of the heart following ligation of the left anterior descending coronary artery. These grafts persisted for 8 weeks and histological analysis demonstrated increased capillary density in the treatment group relative to control [7].

\section{Adult Stem Cell Treatments}

There is a larger evidence base on adult stem cells in vascular regeneration, which has honed in on the endogenous and exogenous utility of vasculogenic EPC's. EPC's are derived from haemangioblasts and reside within osteoblastic and vascular niches. In response to ischaemia, chemochemokines such as Vascular Endothelial Growth Factor (VEGF) activate matrix metalloproteinase enzymes that cleave Kit-ligands and mobilise EPC's. This enables EPC's to home towards ischaemic tissues where they: differentiate into pericytes, smooth muscle, or endothelial-cells; and facilitate angiogenesis by releasing paracrine factors [8]. In contrast to the endogenous utility of EPC's, the exogenous role of EPC's in vascular regeneration is based on transplantation. These approaches will be considered in turn.

Evidence on the targeted mobilization of resident adult stem cells to ischaemic tissue appears promising. Stimulating EPC differentiation, proliferation and migration by modulating the microenvironment of osteoblastic and vascular niches may be a therapeutic strategy for vascular regeneration [9]. In rodent models of ischaemia, adenoviral vectors expressing angiogenic chemokines such as VEGF were observed to promote the mobilisation and recruitment of adult stem cells into neo-angiogenic tissue and accelerate vascular regeneration [10]. However, chemocytokine driven adult stem cell mobilisation may not entirely enhance vascular regeneration. This may, in part, be explained by the inhibitory effect of chemokines such as (Granulocyte Colony Stimulating Factor) G-CSF on Stromal Cell Derived Factor-1a (SDF-1 $\alpha$ ) signalling in myocardial repair. SDF-1 $\alpha$ signalling normally functions to recruit Chemokine Receptor-4 (CXCR4)-expressing adult stem cells to the ischaemic myocardium to facilitate regeneration [11]. However, clinical trials using the chemokine G-CSF for stem cell mobilisation reported negative results in enhancing vascular regeneration and cardiac function possibly due to interruption of GCSF with SDF-1a signalling [12].

The paracrine effects of adult stem cells are critical in enhancing vascular regeneration. In rodent models of transmural myocardial infarction grafted with MSC's the paracrine factors basic Fibroblast Growth Factor (bFGF), VEGF and stem cell homing factor are increased in the peri-infarct zone. The presence of these paracrine factors positively correlated with an increase in capillary density of $\sim 40 \%$ and left ventricular contractility. Furthermore, down-regulation of the pro-apoptotic protein Bax in the peri-infarct zone was reported [13]. These findings suggest the therapeutic potential of adult stem 
Page 3 of 5

cells for vascular regeneration is mediated via paracrine-induced angiogenesis and cytoprotection.

The therapeutic potential of adult stem cells for vascular regeneration has been further harnessed using transplantation strategies. Intracoronary injection of autologous bone marrow derived stem cells was used in the randomised controlled BOne marrOw cell transfer in patients with acute ST-elevation myocardial infarction (BOOST) trial. 60 patients, who were 5-days post ST-elevation myocardial infarction, were randomised to receive autologous bone marrow stem cells or control. Significant increases in (Left Ventricular Ejection Fraction) LVEF were recorded in the treatment group [14]. This was corroborated by a study that reported a significant increase in LVEF and myocardial oxygen consumption following direct myocardial injection of stem cells in 14 patients with end-stage ischaemic cardiomyopathy [15]. Additionally, improvements in LVEF coupled with a reduction in infarct size have been reported by several meta-analyses of trials on patients with coronary disease who received intracoronary stem cell transplantation [16]. Further evidence was provided by the large multi-centre double-blinded randomised controlled Reinfusion of Enriched Progenitor cells And Infarct Remodelling in Acute Myocardial Infarction (REPAIR-AMI trial), which reported: an increase in LVEF 4 months post-adult stem cell transplantation; improvements in vasomotor responsiveness and microvascular function at 4 months; and a significant reduction in recurrent infarction requiring revascularisation at one year [17]. The ongoing large-scale multinational Bone marrow-derived mononuclear stem cells (BM-MNC) on all-cause mortality in Acute Myocardial Infarction (BAMI) trial, involving 3,000 patients, will provide further information on the efficacy of percutaneous trans-coronary infusion of autologous Bone Marrow Mononuclear Cell (BM-MNC) grafting in restoring cardiac function following acute myocardial infarction [18]. This has led onto the world's first surgical clinical trials assessing the efficacy of adult stem cells in cardiac regeneration, which are ongoing and include: the double blind placebo-controlled Transplantation of enriched autologous bone-marrow derived CD133+ cells in patients having coronary surgery after STEMI 1 (TransACT-1) trial; and the randomised-blinded controlled TransACT-2 trial involving bonemarrow derived stem cell transplantation in patients undergoing left ventricular restoration surgery for dilated ischemic end-stage heart failure $[19,20]$.

Evidence on the therapeutic utility of adult stem cells in enhancing vascular regeneration in peripheral arterial disease was demonstrated in the controlled Therapeutic Angiogenesis using Cell Transplantation (TACT) trial. Patients who received intramuscular injection of adult stem cells had improved rest pain, claudication distance and transcutaneous oxygen pressures relative to the control group [21].

However, the evidence on transplantation of adult stem cells to enhance vascular regeneration is limited in its application. For example, the 18 month follow-up data from the BOOST trial failed to show a sustained benefit from single injection of autologous bone marrow derived-stem cells on LVEF relative to standardised care [22]. Additionally, a separate study found that whilst intracoronary grafting of autologous bone marrow cells within 24 hours of optimum reperfusion therapy favourably affected infarct remodelling, it did not augment recovery of global LV function [23]. Leading on from this concerns were raised over the safety of grafted adult stem cells in the randomised controlled Autologous Stem cell Transplantation in Acute Myocardial Infarction (ASTAMI) trial. 24 out of the 49 patients included in the study experienced adverse effects, which included: ischaemic chest pain during intracoronary stem cell transfer; and ventricular fibrillation post-transplantation. The ASTAMI trial failed to demonstrate clinical significance of adult stem cells in improving LVEF [24]. The differences in results between the REPAIR-AMI and ASTAMI trials may be related to the procedure used to culture autologous bone marrow derived-stem cells in the ASTAMI trial, which may have adversely impacted upon their utility. For example, in the ASTAMI trial the variable expression of the cell-surface antigen CXCR4, which is pivotal for the homing of grafted cells to the ischaemic myocardium, may have been influenced by cell storage conditions [25]. Furthermore, the ASTAMI trial was only powered to detect a 5\% increase in LVEF following stem cell engraftment whereas the large REPAIR-AMI trial detected a $2.5 \%$ improvement following stem cell transplantation. Table 1 summarises the clinical trials on stem cell treatments for vasculopathies.

\begin{tabular}{|l|l|l|}
\hline Trial & Nature of vasculopathy & Outcome \\
\hline BOOST & Coronary artery disease & Significant improvements in LVEF following ST elevation myocardial infarction \\
\hline REPAIR-AMI & Coronary artery disease & $\begin{array}{l}\text { Increased LVEF 4 months post-adult stem cell transplantation; improvements in } \\
\text { vasomotor responsiveness and microvascular function at 4 months; and a significant } \\
\text { reduction in recurrent infarction requiring revascularisation at one year }\end{array}$ \\
\hline BAMI & Coronary artery disease & Ongoing trial; results awaited \\
\hline TransACT-1 & Ischaemic cardiomyopathy & Ongoing trial; results awaited \\
\hline TranSACT-2 & Ischaemic cardiomyopathy & Ongoing trial; results awaited \\
\hline TACT & Peripheral vascular disease & $\begin{array}{l}\text { Improved rest pain, claudication distance and transcutaneous oxygen pressures relative } \\
\text { to the control group }\end{array}$ \\
\hline ASTAMI & Coronary artery disease & $\begin{array}{l}\text { No improvement in LVEF; adverse effects included ischaemic chest pain and ventricular } \\
\text { fibrillation post-grafting }\end{array}$ \\
\hline
\end{tabular}

Table 1: Induced Pluripotent Stem Cells Treatments

If and how the heart regenerates after an injury event is highly debated. Research led by Anversa has shown that c-kit expressing cells can produce new cardiomyocytes and may help regenerate damaged cardiac tissue [26]. Anversa and others have helped usher the cells into 
clinical trials. However, recent evidence casts doubt on the credibility of whether c-kit cells can produce new cardiomyocytes. It was reported that endogenous c-kit+ cells did produce new cardiomyocytes within the heart, although at a percentage of approximately 0.03 or less, and if a preponderance towards cellular fusion is considered, the percentage falls to below approximately 0.008 . By contrast, c-kit+ cells amply generated cardiac endothelial cells. Thus, endogenous c-kit+ cells can generate cardiomyocytes within the heart, although probably at a functionally insignificant level [27]. These findings triggered an investigation by the Harvard Medical School and Brigham and Women's Hospital, which determined that the data contained within Anversa's paper was sufficiently compromised that a retraction of their work, published in Circulation 2012, was warranted.

In vitro iPS cells have been shown to differentiate into EC's, cardiomyocytes, smooth muscle cells and vascular mural cells [28]. Existing methods for human iPS cell cardiac differentiation are efficient but require complex, undefined medium constituents that hinder further elucidation of the molecular mechanisms of cardiomyogenesis. Accordingly a new protocol has been developed that provides a chemically defined platform for cardiac specification of human iPS cells that allows the elucidation of cardiomyocyte macromolecular and metabolic requirements, which will provide a minimal system for the study of maturation and subtype specification [29]. Furthermore, a separate study demonstrated that human iPS cells can differentiate into functional cardiomyocytes. Immunocytochemistry of the isolated cardiomyocytes revealed indistinguishable sarcomeric organisations and electrophysiology studies indicated that iPS cells have a capacity like ES cells for differentiation into nodal-, atrial-, and ventricular-like phenotypes based on action potential characteristics. Both the iPS and ES cellderived cardiomyocytes exhibited responsiveness to $\beta$-adrenergic stimulation manifested by an increase in spontaneous rate and a decrease in action potential duration [30]. Leading on from this it has been demonstrated that allogeneic iPS cells injected directly into the ischemic region in a rodent model of myocardial infarction differentiated into EC's, cardiomyocytes and smooth muscle cells thus regenerating cardiac morphology and function [31]. Furthermore, the utility of iPS cells to augment vascular regeneration was demonstrated in a separate study that used cord blood and fibroblast derived-iPS cells to generate vascular EPC's. When injected systemically or directly into the vitreous humour of retinal ischemia-reperfusion-injured adult non-obese diabetic-SCID mice, the iPS cell-derived endothelial progenitor cells were found to home towards, engraft into, and repair the damaged retinal vasculature [32].

However, the clinical utility of iPS cell treatments for vascular regeneration is limited in its application. For example, iPS cells are associated with teratoma formation, which may require immunosuppression in the host. If the iPS cells are sourced autologously there is potential for the genetic or acquired abnormalities that predisposed the patient to the particular disease to be inherent within and thus recapitulated in their iPS cells. Additionally, as a potential pro-angiogenic therapy, iPS cell derivedEC may promote tumor angiogenesis, pathological retinopathy, or neovascularization.

\section{Conclusion}

Stem cell treatments offer a novel curative therapeutic dimension for vascular regeneration. Early preclinical and clinical results are promising; however, further research is required into improving longterm graft survival, refining stem cell differentiation and purification culture methods, safety, and negating the potential oncoplastic transformation of transplanted cells. Overcoming these hurdles along with the results of ongoing large-scale clinical trials will ultimately determine whether the role of stem cells in vascular regeneration is based on hope or expectation.

\section{References}

1. Santulli G (2013) Epidemiology of cardiovascular disease in the 21st century: Updated numbers and updated facts. Journal of Cardiovascular Disease 1: 2326-3121.

2. Singla DK, Hacker TA, Ma L, Douglas PS, Sullivan R, et al. (2006) Transplantation of embryonic stem cells into the infarcted mouse heart: formation of multiple cell types. J Mol Cell Cardiol 40: 195-200.

3. Laflamme MA, Chen KY, Naumova AV, Muskheli V, Fugate JA, et al. (2007) Cardiomyocytes derived from human embryonic stem cells in pro-survival factors enhance function of infarcted rat hearts. Nat Biotechnol 25: 1015-1024.

4. Burridge PW, Keller G, Gold JD, Wu JC (2012) Production of de novo cardiomyocytes: human pluripotent stem cell differentiation and direct reprogramming. Cell Stem Cell 10: 16-28.

5. Nakayama KH, Hou L, Huang NF (2014) Role of extracellular matrix signaling cues in modulating cell fate commitment for cardiovascular tissue engineering. Adv Healthc Mater 3: 628-641.

6. Chong JJ, Yang X, Don CW, Minami E, Liu YW, et al. (2014) Human embryonic-stem-cell-derived cardiomyocytes regenerate non-human primate hearts. Nature 510: 273-277.

7. Leeper NJ, Hunter AL, Cooke JP (2010) Stem cell therapy for vascular regeneration: adult, embryonic, and induced pluripotent stem cells. Circulation 122: 517-526.

8. Asahara T, Kawamoto A (2004) Endothelial progenitor cells for postnatal vasculogenesis. Am J Physiol Cell Physiol 287: C572-579.

9. Mazhari R, Hare JM (2007) Mechanisms of action of mesenchymal stem cells in cardiac repair: potential influences on the cardiac stem cell niche. Nat Clin Pract Cardiovasc Med 4 Suppl 1: S21-26.

10. Rafii S, Heissig B, Hattori K (2002) Efficient mobilization and recruitment of marrow-derived endothelial and hematopoietic stem cells by adenoviral vectors expressing angiogenic factors. Gene Ther 9: 631-641.

11. Ghadge SK, Mühlstedt S, Ozcelik C, Bader M (2011) SDF-1̂̂̃ as a therapeutic stem cell homing factor in myocardial infarction. Pharmacol Ther 129: 97-108.

12. Engelmann MG, Theiss HD, Theiss C, Henschel V, Huber A, et al. (2010) G-CSF in patients suffering from late revascularised ST elevation myocardial infarction: final 1-year-results of the G-CSF-STEMI Trial. Int J Cardiol 144: 399-404

13. Tang YL, Zhao Q, Qin X, Shen L, Cheng L, et al. (2005) Paracrine action enhances the effects of autologous mesenchymal stem cell transplantation on vascular regeneration in rat model of myocardial infarction. Ann Thorac Surg 80: 229-236.

14. Wollert KC, Meyer GP, Lotz J, Ringes-Lichtenberg S, Lippolt P, et al. (2004) Intracoronary autologous bone-marrow cell transfer after myocardial infarction: the BOOST randomised controlled clinical trial. Lancet 364: 141-148.

15. Perin EC, Dohmann HF, Borojevic R, Silva SA, Sousa AL, et al. (2003) Transendocardial, autologous bone marrow cell transplantation for severe, chronic ischemic heart failure. Circulation 107: 2294-2302.

16. Henning RJ (2011) Stem cells in cardiac repair. Future Cardiol 7: 99-117.

17. Mills JS, Rao SV (2007) REPAIR-AMI: stem cells for acute myocardial infarction. Future Cardiol 3: 137-140.

18. Clifford DM, Fisher SA, Brunskill SJ, Doree C, Mathur A, et al. (2012) Long-term effects of autologous bone marrow stem cell treatment in 
Citation: Seewoodhary J (2014) Novel Approaches to Vasculopathies: The Role of Stem Cells and Regenerative Medicine. J Cardiovasc Dis Diagn 2: 166. doi:10.4172/2329-9517.1000166

Page 5 of 5

acute myocardial infarction: factors that may influence outcomes. PLoS One 7: e37373.

19. Ascione R, Madeddu P (2009) Risk and benefit of CD133+ progenitors. Circ Res 105: e2.

20. http://cteu.bris.ac.uk/trials/

21. Matoba S, Tatsumi T, Murohara T, Imaizumi T, Katsuda Y, et al. (2008) Long-term clinical outcome after intramuscular implantation of bone marrow mononuclear cells (Therapeutic Angiogenesis by Cell Transplantation $[\mathrm{TACT}]$ trial) in patients with chronic limb ischaemia. Am Heart J 156: 1010-1018.

22. Meyer GP, Wollert KC, Lotz J, Steffens J, Lippolt P, et al. (2006) Intracoronary bone marrow cell transfer after myocardial infarction eighteen months' follow-up data from the randomised, controlled BOOST (BOne marrOw transfer to enhance ST-elevation infarct regeneration) trial. Circulation 113: 1287-1294.

23. Janssens S, Dubois C, Bogaert J, Theunissen K, Deroose C, et al. (2006) Autologous bone marrow-derived stem-cell transfer in patients with STsegment elevation myocardial infarction: double-blind, randomised controlled trial. Lancet 367: 113-121.

24. Lunde K, Solheim S, Aakhus S, Arnesen H, Abdelnoor M, et al. (2005) Autologous stem cell transplantation in acute myocardial infarction: The ASTAMI randomised controlled trial. Intracoronary transplantation of autologous mononuclear bone marrow cells, study design and safety aspects. Scand Cardiovasc J 39: 150-158.

25. Seeger FH, Tonn T, Krzossok T, Zeiher AM, Dimmeler S (2007) Cell isolation procedures matter: a comparison of different isolation protocols of bone marrow mononuclear cells used for cell therapy in patients with acute myocardial infarction [Abstract 1680]. Eur Heart J 28: 766-772.

26. Kajstura J, Rota M, Cappetta D, Ogórek B, Arranto C, et al. (2012) Cardiomyogenesis in the aging and failing human heart. Circulation 126: 1869-1881.

27. van Berlo JH, Kanisicak O2, Maillet M3, Vagnozzi RJ3, Karch J3, et al. (2014) c-kit+ cells minimally contribute cardiomyocytes to the heart. Nature 509: 337-341.

28. Narazaki G, Uosaki H, Teranishi M, Okita K, Kim B, et al. (2008) Directed and systematic differentiation of cardiovascular cells from mouse induced pluripotent stem cells. Circulation 118: 498-506.

29. Burridge PW, Matsa E, Shukla P, Lin ZC, Churko JM, et al. (2014) Chemically defined generation of human cardiomyocytes. Nat Methods .

30. Zhang J, Wilson GF, Soerens AG, Koonce CH, Yu J, et al. (2009) Functional cardiomyocytes derived from human induced pluripotent stem cells. Circ Res 104: e30-41.

31. Li Z, Wu JC, Sheikh AY, Kraft D, Cao F, et al. (2007) Differentiation, survival, and function of embryonic stem cell derived endothelial cells for ischemic heart disease. Circulation 116: I46-54.

32. Parks TS, Bhutto I, Zimmerlin L, Huo JS, Nagaria P, et al. (2014) Vascular progenitors from cord blood-derived induced pluripotent stem cells possess augmented capacity for regenerating ischemic retinal vasculature. Circulation 129: 359-372. 